\title{
Presentation of neuroendocrine self in the thymus: a necessity for integrated evolution of the immune and neuroendocrine systems
}

\author{
Vincent Geenen \\ University of Liege, GIGA-Research Center of Immunoendocrinology, Sart Tilman, Belgium \\ Address for correspondence: Vincent Geenen, M.D., Ph.D., University of Liege, GIGA-Research Center of \\ Immunoendocrinology, CHU-B34, B-4000 Liege-Sart Tilman, Belgium. vgeenen@ulg.ac.be
}

\begin{abstract}
During evolution, from ancestor thymoids scattered in gill baskets of the lamprey, the first unique thymus appeared in jawed cartilaginous fishes around 450-500 millions years ago, concomitantly or shortly after the emergence of recombinase-dependent adaptive immunity. The major biological function of the thymus is to generate a diverse repertoire of $\mathrm{T}$ cell receptors that are self tolerant. The thymus achieves this role by using two complementary and intimately associated mechanisms: apoptotic deletion of $T$ cell clones bearing a TCR with high affinity for self-antigens presented by MHC proteins on thymic epithelial cells (TECs) and dendritic cells (DCs); and generation of self-antigen-specific natural regulatory $\mathrm{T}\left(\mathrm{nT}_{\text {reg }}\right)$ cells. Moreover, the escape from thymic central self-tolerance plays a primary role in the development of autoimmune diseases that are a significant burden for the quality of life and health-care cost. Our new knowledge in thymus physiology and physiopathology is currently translated into innovative therapeutic strategies against these devastating chronic diseases.
\end{abstract}

Keywords. thymus; antigen presentation; central self-tolerance; autoimmunity; AIRE; type 1 diabetes; IGF-2; Graves' disease

\section{The moving place of the thymus in the history of medicine}

Claude Galen (129-199 or 217 AD), one of the "fathers" of medicine, and Hippocrates (ca. 460 BCca. $370 \mathrm{BC}$ ), first reported the observation of the thymus that he so named because of its close resemblance with the leaf of the plant Thymus vulgaris. Galen suspected the thymus to be the seat of soul, humor, eagerness, and fortitude. Given Galen's strong influence on Western medicine until the 18th century, this old misconception most probably explains why the words thymie and troubles thymiques still resonate in French medical language mood and mood disorders that are observed in neuropsychiatric diseases. Jacopo Berengario da Carpi (14601530) then provided the first complete anatomical description of the thymus.

For a long period of time, the thymus was thought to be a vestigial organ that had become useless and redundant during phylogeny and ontogeny after puberty. In the early 1900s, J. August Hammar in Sweden highlighted the important neuroendocrine regulation of the thymus, in particular, the relationship between thymic hyperplasia and acromegaly, Graves' disease, and castration. ${ }^{1}$ The thymus was then considered a gland and an intrinsic component of the endocrine system. However, despite the identification of several thymic "hormones," the model of endocrine cell-to-cell signaling failed to characterize the complex molecular dialogue between thymic stromal cells and thymic T cells (thymocytes). In 1959 and 1961, after Jacques F.A.P. Miller demonstrated the crucial role of the thymus in mouse leukemia and $\mathrm{T}$ cell development, ${ }^{2,3}$ while the endocrine feature of the thymus progressively vanished until the discovery of the intrathymic transcription of neuroendocrine genes. 


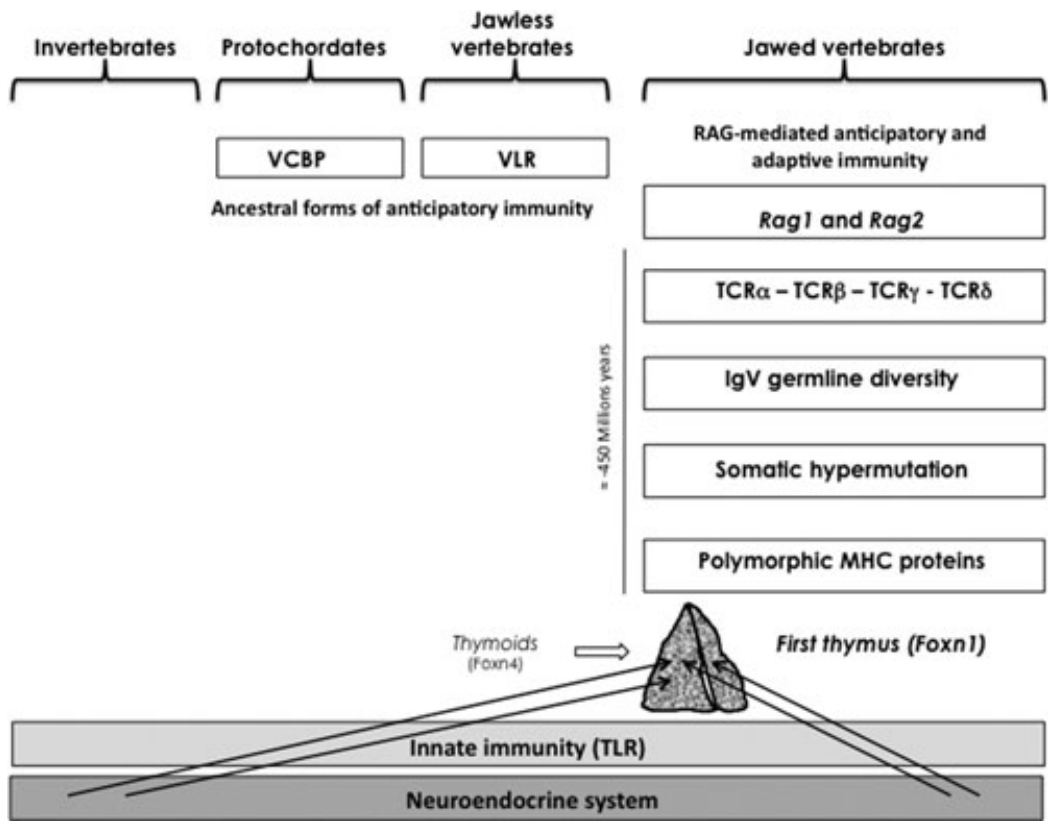

Figure 1. Integrated evolution of the immune and neuroendocrine systems. Essential components of the neuroendocrine system were established long ago and did not display important variation during evolution except for gene duplication and differential RNA splicing. The appearance of RAG-dependent adaptive immunity in jawed vertebrates was associated with a high risk of autotoxicity directed against the neuroendocrine system. Of note, from ancestor lamprey thymoids, the first unique thymus emerged concomitantly in jawed cartilaginous fishes, and the intrathymic presentation of neuroendocrine-related genes (arrows) may be viewed a posteriori as a very efficient and economical way to instruct the adaptive $\mathrm{T}$ cell system to tolerate neuroendocrine antigens as early as during intrathymic T cell development and differentiation. VCBP, variable-region-containing chitin-binding protein; VLR, variable lymphocyte receptor.

\section{Emergence of the thymus in evolution}

In all living species, the neuroendocrine and innate immune systems have evolved in parallel and still coexist today without any problem (Fig. 1). Indeed, Toll-like receptors (TLR), which are the most important mediators of innate immunity, do not have the ability to react against normal and undamaged self. Some 450-500 million years ago, the emergence of transposon-like recombination-activating genes Rag1 and Rag2 in jawed fishes (sharks and rays) promoted the development of adaptive immunity. ${ }^{4-6}$ The appearance of these elements in the genome of gnathostomes, and the subsequent development of the combinatorial immune system, has been sometimes described as to the "Big Bang" of immunology. Gene recombination in somatic lymphoid cells is responsible for the random generation of the extreme diversity of immune receptors for antigens, B cell (BCR) and T cell receptors (TCR). Because of its inherent autotoxic- ity, the emergence of this new sophisticated type of immune response exerted an evolutive pressure so strong that, in accordance with Paul Ehrlich's prediction of horror autotoxicus, novel structures and mechanisms appeared with the specific role of establishing protection against potential autoimmune attacks to the host (immunological self-tolerance). Of note, the first unique thymus also appeared in the jawed cartilaginous fishes, but was preceded by thymus-like lymphoepithelial structures in the gill baskets of lamprey larvae, as recently demonstrated. ${ }^{7}$ These structures named thymoids express the gene-encoding forkhead box N4 (Foxn4), the orthologue of Foxn1, the transcription factor responsible for the differentiation of thymic epithelium in higher vertebrates. Thus, FOXN1 stands at a crucial place in the emergence of thymus epithelium that is an absolute requirement for the control of $\mathrm{T}$ cell differentiation and central self-tolerance induction. $^{8}$ 


\section{Presentation of self in the thymus}

Self-antigen presentation by major histocompatibility complex (MHC) proteins on thymic stromal cells (epithelial cells (TECs) and dendritic cells (DCs), mainly) is the central mechanism determining the process of T cell differentiation, which includes three alternative and exclusive fates for developing thymocytes: negative selection of self-reactive T cells generated during the random generation of TCR diversity, catalyzed by recombination-activating enzymes RAG1 and RAG2; selection of self-specific natural regulatory $\left(\mathrm{nT}_{\text {reg }}\right)$ cells; and survival and positive selection of $\mathrm{CD}^{+}{ }^{+}$and $\mathrm{CD} 8^{+}$effector and self-tolerant $\mathrm{T}$ cells. The first two events ensure the establishment of the thymus-dependent central arm of immunological self-tolerance, while the avidity/affinity of the TCR-self-antigen-MHC interaction is the central determinant of $\mathrm{T}$ cell negative or positive selection. One important unresolved question, however, is how the same MHC-self-antigen complexes are able to mediate negative selection of self-reactive $\mathrm{T}$ cells and yet generate self-specific $\mathrm{nT}_{\text {reg }}$ cells (extensively discussed in Ref. 9).

Another question has long concerned the nature of self that is presented in the thymus to differentiating $\mathrm{T}$ cells, in particular during fetal life. Since its original formulation by Frank M. Burnet, self has been a seminal word, first coined in the immunological language as a fecund metaphor with some equivocal correlations to the neurocognitive sciences and even philosophy. The precise identity of self was not elucidated before a series of studies initiated in the late 1980s and 1990s. ${ }^{10-16}$ Our personal contribution to this field was to define the biochemical nature of the neuroendocrine self. First, thymic neuroendocrine self-antigens correspond to peptide sequences that are mostly conserved throughout evolution of their related family. Second, a hierarchy and an economic principle characterize their profile of expression in the thymus, as one dominant member per family is synthesized in TECs: that is, oxytocin (OT) for the neurohypophysial family, neurokinin A for tachykinins, neurotensin for neuromedins, corticostatin for somatostatins, and insulin-like growth factor 2 (IGF-2) for the insulin family. This hierarchy is very important because the strength of immunological tolerance to a protein/peptide is proportional to its intrathymic concentration. ${ }^{17}$ Third, the autoimmune regulator gene/protein (AIRE) controls the intrathymic transcription of most of the genes encoding neuroendocrine self-antigens. ${ }^{18}$ Following AIRE-regulated gene transcription, thymic neuroendocrine precursors are not processed according to the classic model of neuroendocrine secretion but as antigens for presentation by, or in association with, thymic MHC proteins. ${ }^{14}$ Fourth, according to the cryptocrine model of cell-to-cell signaling, ${ }^{19}$ thymic $\mathrm{T}$ cells express functional neuroendocrine cognate receptors. ${ }^{20}$ For example, binding of thymic OT to the OT receptor expressed by thymic preT cells phosphorylates focal adhesion kinases, ${ }^{21}$ and this might promote the establishment of immunological synapses between TECs and T cells. Finally, for some neuroendocrine-related precursors, their transcription in TECs precedes their eutopic expression in peripheral neuroendocrine glands/cells, ${ }^{20}$ and this is also very relevant with regard to the induction of self-tolerance to neuroendocrine principles. Therefore, depending on their behavior as the source of self-antigens or cryptocrine ligands, respectively, the thymic repertoire of neuroendocrinerelated precursors transposes at the molecular level the multiple roles of the thymus in T cell differentiation (Fig. 2). ${ }^{22,23}$

The organization of the thymic repertoire of neuroendocrine self-precursors is also significant from an evolutionary point of view. Because neuroendocrine hormones were implicated in the regulation of many physiological functions before the appearance of the anticipatory adaptive immune response, they had to be protected from the risk of autoimmunity inherent to this type of immune lottery. The hypothalamic peptide OT controls different steps of the reproductive process, starting from social affiliation and bonding to parturition and lactation. ${ }^{24}$ Consequently, self-tolerance toward OT is important for the preservation of animal and human species. Via its dominant expression in TECs, OT is more tolerated than its homologue vasopressin, which essentially controls water balance and vascular tone. Of note, rare cases of autoimmune diabetes insipidus have been reported, ${ }^{25-27}$ whereas autoimmunity toward hypothalamic OT-ergic neurons have never been described. With regard to the insulin family, no autoimmunity has been described against IGF-2, the dominant self-antigen of the insulin family during fetal life, whereas insulin is the primary autoantigen of type 1 diabetes 


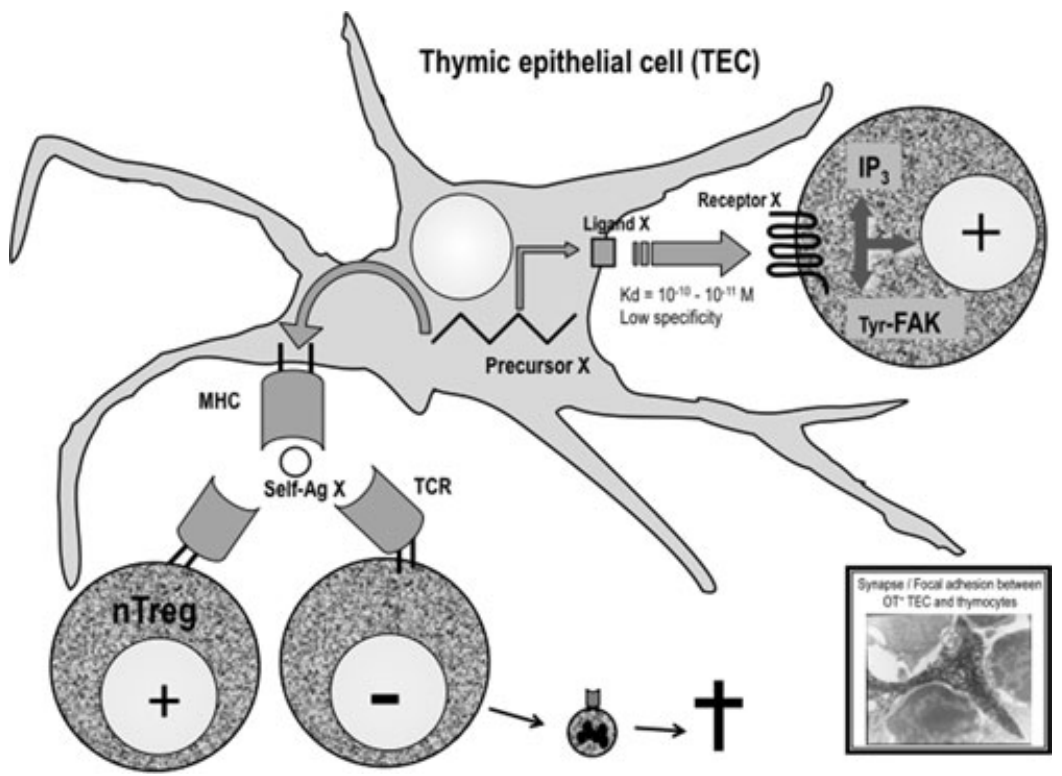

Figure 2. The role of thymic neuroendocrine precursors in $\mathrm{T}$ cell differentiation. A precursor $\mathrm{X}$ encoded by a neuroendocrinerelated gene in a TEC is the source of two distinct types of signaling with thymocytes. First, it delivers a cryptocrine ligand $\mathrm{X}$ that is not secreted but targeted at the outer surface of TEC plasma membrane. Through direct membrane-to-membrane contact, this ligand binds with high affinity to a cognate neuroendocrine receptor expressed by thymocytes. For example, OT-mediated cryptocrine signaling activates phosphoinositide turnover with an increase of $\mathrm{IP}_{3}$ in pre-T cells and phosphorylates focal adhesionrelated kinases, which may promote the formation of synapses between TEC and thymocytes. Second, the same precursors may be processed for presentation of neuroendocrine self-epitopes by thymic MHC proteins. Deletion of T cell clones bearing a TCR specific for MHC-neuroendocrine self-antigen complexes, together with generation of self-antigen-specific $\mathrm{nT}_{\text {reg, }}$, is responsible for the establishment of central self-tolerance toward neuroendocrine gene/protein families. How precisely the same MHC-self-antigen complexes are able to delete self-reactive $\mathrm{T}$ cells and select self-specific $\mathrm{nT}_{\text {reg }}$ cells remains a major unsolved question. FAK, focal adhesion kinase; $\mathrm{IP}_{3}$, inositol triphosphate.

(T1D). ${ }^{28}$ However, through cross-tolerance, thymic neuroendocrine self-antigens promote selftolerance to all the homologous members of their family as evidenced by the weaker tolerance to insulin in $I g f 2^{-1-}$ than in normal mice. $^{29}$

\section{The escape from central self-tolerance as a primary event in autoimmunity, and the concept of negative self-vaccination}

As already theorized by Burnet, the pathogenesis of autoimmune diseases may first depend on a failure of self-tolerance and the development of "forbidden" self-reactive immune clones. ${ }^{30}$ The progressive increase in immune complexity during evolution is associated with a higher incidence of self-tolerance failures, most of them occurring in the human species. There is more and more evidence that a thymus dysfunction in the establishment of central self-tolerance drives the development of the autoimmune response toward many organs. Thymus transplantation from nonobese diabetic (NOD) mice, an animal model of T1D, was shown to induce diabetes in normal recipients. ${ }^{31}$ Igf2 2 transcription is deficient in the thymus of diabetes-prone BioBreeding (DPBB) rats, another animal model of T1D, such a defect might contribute to both the absence of tolerance toward $\beta$ cells and the usual lymphopenia (including $\mathrm{RT}^{+}{ }^{+} \mathrm{T}_{\text {reg }}$ cells) observed in these animals. ${ }^{32}$ Mice with a thymus-restricted insulin defect develop strong proinsulin-specific $\mathrm{T}$ cell reactivity, ${ }^{33}$ and thymus-specific deletion of insulin induces rapid development of an autoimmune diabetes. ${ }^{34}$ Nevertheless, despite the current evidence for a role of thymic insulin in the induction of $\beta$ cell tolerance (even at a very low level of transcription in medullary TECs), it is important to note that insulin per se failed to restore self-tolerance toward $\beta$ cells in all animal or clinical trials to date.

Loss-of-function AIRE single mutations are responsible for a very rare autosomal recessive disease named autoimmune polyendocrinopathy, 
candidiasis, and ectodermal dystrophy or autoimmune polyglandular syndrome type 1 . Depending on their genetic background, Aire ${ }^{-/-}$mice exhibit several signs of peripheral autoimmunity, which are associated with a significant decrease in the intrathymic transcription level of neuroendocrine genes, including those encoding OT, proinsulin 2, and IGF-2. ${ }^{18,35}$ Of note, with regard to autoimmune thyroiditis, which is the most frequent autoimmune disease, all major thyroid-related antigens (thyroperoxydase, thyroglobulin, and thyrotropin receptor (TSHR)) are also transcribed in TECs in normal conditions. ${ }^{15,36}$ Thymic hyperplasia is commonly observed in Graves' disease, ${ }^{37}$ and it was recently shown that homozygotes for an SNP allele predisposing to Graves' disease have significantly lower intrathymic TSHR transcripts than carriers of the protective allele. ${ }^{38}$ Another credit to a defective central tolerance driving the development of autoimmunity was recently provided with the demonstration of the central role played by a defect in intrathymic $\alpha$-myosin expression in the pathogenesis of autoimmune myocarditis in mice and humans. ${ }^{39}$ Our current in-depth knowledge of thymus physiology and physiopathology has now been translated into the design of innovative tolerogenic and regulatory strategies aimed at restoring central selftolerance that is absent or defective in autoimmunity. ${ }^{40-42}$ The concept of negative self-vaccination has been proposed and is based both on the competition between thymic self-antigens and peripheral target antigens for presentation by MHC proteins, as well as a tolerogenic response-including recruitment of $\mathrm{T}_{\text {reg }}$ cells - induced by MHC presentation of thymic self-epitopes. ${ }^{43,44}$ With this perspective, a research consortium in Wallonia is working on the development of a negative/tolerogenic self-vaccination with the thymic self-peptides related to T1D (Tolediab project).

\section{Thymus involution and immunosenescence}

Although thymopoiesis is maintained until late in life, ${ }^{45-47}$ thymus involution remains the hallmark of immunosenescence that is characterized by a higher susceptibility to infections, as well as a decrease in vaccine and antitumor immune responses. Thymic fat and fibrous involution is associated with a marked decrease in the generation of diverse $\mathrm{T}$ cells (in particular, naive $\mathrm{CD} 4^{+} \mathrm{T}$ cells), an expan- sion of memory $\mathrm{CD}^{+} \mathrm{T}$ cells, and a diminished influence of central self-tolerance. Involution of the thymus after hypophysectomy was early evidence for the control of the thymus function by a neuroendocrine gland. ${ }^{48}$ Since then, numerous studies have unambiguously demonstrated that the hypophysial growth hormone $(\mathrm{GH})$ is able to reverse the age-dependent involution of the thymus. ${ }^{4-51}$ Intrathymic proliferation of $\mathrm{T}$ cell precursors and thymic output of naive $\mathrm{T}$ cells are significantly decreased in adults with GH deficiency, and GH replacement restores these two parameters. ${ }^{52}$ Today, restoration of thymus function appears more and more to be an important objective in the elderly, as well as in patients suffering with acquired immunodeficiency syndrome or several hematological diseases. ${ }^{53,54}$ It can be anticipated that GH, IGF-1, $\mathrm{GH}$ secretagogues (such as ghrelin), GH and ghrelin receptor agonists, as well as other thymus-specific growth factors will be used in the near future for regenerating thymopoiesis and, secondarily, immune functions, including response to vaccines in aged and other immunocompromised patients.

\section{Conclusion}

As evidenced in this short overview, a novel era is now beginning for a quantifiable clinical investigation of thymus function in the context of a series of immune-mediated and infectious diseases. Furthermore, pharmacological manipulation of thymusdependent thymopoietic and tolerogenic functions can now be exploited to provide the scientific community with innovative strategies in the treatment of a large number of immune-mediated disorders.

\section{Acknowledgments}

These studies have been supported by the Fund of Scientific Research (F.R.S.-FNRS, Belgium), the Fund for Research in Industry and Agronomy (FRIA, Belgium), the Fund Leon Fredericq for biomedical research at the University Hospital of Liege, the Special Research Fund of the University of Liege, Wallonia (Tolediab, Senegene, ThymUP, and Raparray projects), the Belgian Association of Diabetes, an Independent Research Grant (Pfizer Europe), the European Commission (Eurothymaide FP6 Integrated Project, www.eurothymaide.org), the Juvenile Diabetes Research Foundation (JDRF, New York), and the European Federation for the Study of Diabetes (EFSD, Düsseldorf). 


\section{Conflicts of interest}

The author declares no conflicts of interest.

\section{References}

1. Hammar, J.A. 1921. The new views as to the morphology of the thymus gland and their bearing on the problem of the function of the thymus. Endocrinology 5: 543-573.

2. Miller, J.F.A.P. 1959. Role of the thymus in murine leukaemia. Nature 183: 1069.

3. Miller, J.F.A.P. 1961. Immunological function of the thymus. Lancet 2: 748-749.

4. Agrawal, A., Q.M. Eastman \& D.G. Schatz. 1998. Transposition mediated by RAG1 and RAG2 and its implications for the evolution of the immune system. Nature 394: 744-751.

5. Boehm, T. \& C. Bleul. 2007. The evolutionary history of lymphoid organs. Nat. Immunol. 8: 131-135.

6. Hirano, M., S. Das, P. Guo \& M.D. Cooper. 2011. The evolution of adaptive immunity in vertebrates. Adv. Immunol. 109: 125-157.

7. Bajoghli, B., P. Guo, N. Aghaallaei, et al. 2011. A thymus candidate in lampreys. Nature 470: 90-95.

8. Boehm, T. 2011. Design principles of adaptive immune systems. Nat. Rev. Immunol. 11:307-317.

9. Klein, L., M. Hinterberger, G. Wirnsberger \& B. Kyewski. 2009. Antigen presentation in the thymus for positive selection and central tolerance induction. Nat. Rev. Immunol. 9: 833-844.

10. Geenen, V., J.J. Legros, P. Franchimont, et al. 1986. The neuroendocrine thymus: coexistence of oxytocin and neurophysin in the human thymus. Science 232: 508-511.

11. Ericsson, A.E., V. Geenen, F. Robert, et al. 1990. Expression of preprotachykinin-A and neuropeptide-Y in mRNA of the thymus. Mol. Endocrinol. 4: 1211-1218.

12. Geenen, V., I. Achour, F. Robert, et al. 1993. Evidence that insulin-like growth factor 2 (IGF-2) is the dominant thymic member of the insulin superfamily. Thymus 21: 115-127.

13. Jolicœur, C., D. Hanahan \& K.M. Smith. 1994. T cell tolerance toward a transgenic beta-cell antigen and transcription of endogenous pancreatic genes in thymus. Proc. Natl. Acad. Sci. U.S.A. 91: 6707-6711.

14. Vanneste, Y., A. Ntodou-Thome, E. Vandersmissen, et al. Identification of neurotensin-related peptides in human thymic epithelial cell membranes and relationship with major histocompatibility complex class I molecules. J. Neuroimmunol. 76: 161-166.

15. Sospedra, M., X. Ferrer-Francesch, O. Dominguez, et al. 1998. Transcription of a broad range of self-antigens in the thymus suggests a role for central mechanisms in tolerance toward peripheral antigens. J. Immunol. 161: 5918-5929.

16. Derbinski, J., A. Schulte, B. Kyewski \& L. Klein. 2001. Promiscuous gene expression in medullary thymic epithelial cells mirrors the peripheral self. Nat. Immunol. 2: 1032-1039.

17. Ashton-Rickardt, P., A. Bandeira, J.R. Delaney, et al. 1994. Evidence for a differential avidity model of $\mathrm{T}$ cell selection in the thymus. Cell 74: 651-663.

18. Anderson, M.S., E.S. Venanzi, L. Klein, et al. 2002. Projection of an immunological self shadow in the thymus by the Aire protein. Science 298: 1395-1401.
19. Funder, J.W. 1990. Paracrine, cryptocrine, acrocrine. Mol. Cell Endocrinol. 70: C21-C24.

20. Hansenne, I., G. Rasier, C. Pequeux, et al. 2005. Ontogenesis and functional aspects of oxytocin and vasopressin gene expression in the thymus network. J. Neuroimmunol. 158: $67-75$.

21. Martens, H., O. Kecha, C. Charlet-Renard, et al. 1998. Neurohypophysial peptides stimulate the phosphorylation of pre-T cell focal adhesions kinases. Neuroendocrinology 67: 282-289.

22. Geenen, V., B. Goxe, H. Martens, et al. 1995. Cryptocrine signaling in the thymus network and $\mathrm{T}$ cell education to neuroendocrine self-antigens. J. Mol. Med. 73: 449-455.

23. Martens, H., B. Goxe \& V. Geenen. 1996. The thymic repertoire of neuroendocrine-related self-antigens: Physiological implications in T cell life and death. Immunol. Today 17: 312-317.

24. Gimpl, G. \& F. Fahrenholz. 2001. The oxytocin receptor system: structure, function, and regulation. Physiol. Rev. 81: 629-683.

25. Scherbaum, W.A. \& G.F. Bottazzo. 1983. Autoantibodies to vasopressin cells in idiopathic diabetes insipidus: evidence for an autoimmune variant. Lancet 1: 897-901.

26. Imura, H., K. Nakao, A. Shimatsu, et al. Lymphocytic infundibuloneurohypophysitis as a cause of central diabetes insipidus. N. Engl. J. Med. 239: 683-689.

27. De Bellis, A., A. Bizzaro \& A. Bellastella. 2004. Autoimmune central diabetes insipidus. In Immunoendocrinology in Health and Disease. V. Geenen \& G.P. Chrousos, Eds.: 439-459. Marcel Dekker. New York.

28. Nakayama, M., N. Abiru, N. Moriyama, et al. 2005. Prime role for an insulin epitope in the development of type 1 diabetes in mice. Nature 435: 220-223.

29. Hansenne, I., C. Charlet-Renard, R. Greimers \& V. Geenen. 2006. Dendritic cell differentiation and tolerance to insulin-related peptides in Igf2-deficient mice. J. Immunol. 176: 4651-4657.

30. Burnet, F.M. 1973. A reassessment of the forbidden clone hypothesis of autoimmune diseases. Aust. J. Exp. Biol. Med. 50: 1-9.

31. Georgiou, H.M. \& T.E. Mandel. 1995. Induction of insulitis in athymic (nude) mice. The effect of NOD thymus and pancreas transplantation. Diabetes 44: 49-59.

32. Kecha-Kamoun, O., I. Achour, H. Martens, et al. 2001. Thymic expression of insulin-related genes in an animalmodel of type 1 diabetes. Diab. Metab. Res. Rev. 17: 146-152.

33. Chentoufi, A. \& C. Polychronakos. 2002. Insulin expression levels in the thymus modulate insulin-specific autoreactive T cell tolerance: the mechanism by which the IDDM2 locus may predispose to diabetes. Diabetes 41: 1383-1390.

34. Fan, Y., W.A. Rudert, H. Grupillo, et al. 2009. Thymusspecific deletion of insulin induces autoimmune diabetes. EMBO J. 28: 2812-2824.

35. Ramsey, C., O. Winqvist, M. Puhakka, et al. Aire-deficient mice develop multiple features of APECED phenotype and show altered immune response. Hum. Mol. Genet. 11: 397409.

36. Paschke, R. \& V. Geenen. 1995. Messenger RNA expression for a TSH receptor variant in the thymus of a two-year old child. J. Mol. Med. 73: 577-580. 
37. Murakami, M., Y. Hosoi, T. Negishi, et al. 1996. Thymic hyperplasia in patients with Graves' disease. Identification of thyrotropin receptors in human thymus. J. Clin. Invest. 98: 2228-2234.

38. Colobran, R., M. del Pilar Armengol, R. Faner, et al. 2011. Association of an SNP with intrathymic transcription of TSHR and Graves' disease: a role for defective thymic tolerance. Hum. Mol. Genet. 20: 3415-3423.

39. Lv, H., E. Havari, S. Pinto, et al. 2011. Impaired thymic tolerance to $\alpha$-myosin directs autoimmunity to the heart in mice and humans. J. Clin. Invest. 21: 15611573.

40. Geenen, V., M. Mottet, O. Dardenne, et al. 2010. Thymic self-antigens for the design of a negative/tolerogenic selfvaccination gainst type 1 diabetes. Curr. Opin. Pharmacol. 10: 461-472.

41. Chentoufi, A. \& V. Geenen. 2011. Thymic self-antigen expression for the design of a negative/tolerogenic selfvaccination against type 1 diabetes. Clin. Dev. Immunol. doi:10.11555/2011/349368.

42. Daniel, C., B. Weigmann, R. Bronson \& H. von Boehmer. 2011. Prevention of type 1 diabetes in mice by tolerogenic vaccination with a strong insulin mimetope. J. Exp. Med. 208: 1501-1510.

43. Geenen, V. 2006. Thymus-dependent T cell tolerance of neuroendocrine functions. Principles, reflections, and implications for tolerogenic/negative self-vaccination. Ann. N.Y. Acad. Sci. 1088: 284-296.

44. Geenen, V., C. Louis, H. Martens \& The Belgian Diabetes Registry. 2004. An insulin-like growth factor 2-derived selfantigen inducing a regulatory cytokine profile after presentation to peripheral blood mononuclear cells from DQ8+ type 1 diabetic adolescents: preliminary design of a thymus- based tolerogenic self-vaccination. Ann. N.Y. Acad. Sci. 1037: 59-64.

45. Douek, D.C., R.D. McFarland, P.H. Keiser, et al. 1998. Changes in thymic function with age and during the treatment of HIV infection. Nature 396: 690-695.

46. Poulin, J.F., J.M. Viswanathan, J.M. Harris, et al. 1999. Direct evidence for thymic function in adult humans. J. Exp. Med. 190: 479-486.

47. Geenen, V., J.F. Poulin, M.L. Dion, et al. 2003. Quantification of $\mathrm{T}$ cell receptor rearrangement excision circles to estimate thymic function: an important new tool for endocrineimmune physiology. J. Endocrinol. 176: 305-311.

48. Smith, P. 1930. The effect of hypophysectomy upon the involution of the thymus in rat. Anat. Rec. 47: 119-143.

49. Kelley, K.W., D.A. Weigent \& R. Kooijman. 2007. Protein hormones and immunity. Brain Behav. Immun. 21: 384392.

50. Savino, W. \& M. Dardenne. 2000. Neuroendocrine control of thymus physiology. Endocr. Rev. 21: 412-443.

51. Taub, D.D., W.J. Murphy \& D.L. Longo. 2010. Rejuvenation of the aging thymus: growth hormone-mediated and ghrelin-mediated signaling pathways. Curr. Opin. Pharmacol. 10: 408-424.

52. Morrhaye, G., H. Kermani, J.J. Legros, et al. 2009. Impact of growth hormone (GH) deficiency and GH replacement upon thymus function in adult patients. PLoS ONE 4: e5668.

53. Napolitano, L.A., D. Schmidt, M.B. Gotway, et al. 2008. Growth hormone enhances thymic function in HIV-1infected patients. J. Clin. Invest. 118: 1085-1098.

54. Castermans, E., M. Hannon, J. Durieux, et al. 2011. Thymic recovery after allogeneic hematopoietic cell transplantation with non-myeloablative conditioning is limited to patients younger than 60 years of age. Haematologica 96 : 298-306. 